\title{
ESTIMAÇÃO DO NÚMERO DE SALÁRIOS MÍNIMOS ATRIBUÍDO A CADA PROFISSÃO EM FUNÇÃO DE SEU PRESTÍGIO
}

Fátima Aparecida Emm Faleiros Sousa*

José Aparecido da Silva**

SOUSA, F.A.E.F.; SILVA, J.A.da. Estimação do número de salários mínimos atribuído a cada profissão em função de seu prestígio. Rev.latino-am.enfermagem, Ribeirão Preto, v. 8, n. 2, p. 106-110, abril 2000.

O propósito deste estudo foi verificar a relação entre o prestígio profissional escalonado através de estimativas numéricas e o prestígio profissional escalonado através de estimativas do número de salários mínimos atribuído a cada profissão em função de seu prestígio profissional na sociedade. Os resultados mostraram: 1 - a relação entre as estimativas de magnitudes e as estimativas do número de salários mínimos atribuído a cada profissão em função de seu prestígio é caracterizada por uma função de potência com um expoente menor que 1,0. 2 - as ordenações dos graus de prestígio das profissões resultantes de diferentes experimentos envolvendo diferentes amostras de sujeitos são altamente concordantes ( $W=0,85 ; p<0,001)$, levandose em conta que a modalidade utilizada foi a numérica (estimação de magnitudes e de salários mínimos).

\section{UNITERMOS: psicofisica}

Em estudos de FALEIROS SOUSA (1993); FALEIROS SOUSA \& DA SILVA (1996) foi investigado o prestígio profissional de diferentes profissões, entre elas a de Enfermeiro, por meio de diferentes métodos psicofísicos escalares. O método de estimação de magnitude numérica foi empregado em todos os estudos para gerar uma escala de razão de prestígio profissional. A concordância entre os graus de prestígios resultantes deste método utilizado foi altamente significativa, tal como indicada pelo coeficiente de concordância de Kendall (W), corrigido para empates, igual a $0,86\left(\mathrm{x}^{2}=\right.$ 51,$40 ; \mathrm{gl}=12 ; \mathrm{p}(0,001)$. Este valor, portanto, indica que as ordenações dos graus de prestígios profissionais julgadas através do método de estimação de magnitudes numéricas são similares, isto é, independentes das amostras de sujeitos empregados.

No presente estudo, diferentemente dos anteriores, os sujeitos deram estimativas do número de salários mínimos atribuídos a cada profissão em função do prestígio profissional que cada uma delas possui em nossa sociedade. O propósito foi verificar a relação entre o prestígio profissional escalonado através de estimativas numéricas e o prestígio profissional escalonado através de estimativas do número de salários mínimos atribuído a cada profissão em função de seu prestígio profissional na sociedade. O importante deste experimento é que estamos comparando duas escalas genuinamente subjetivas de prestígio profissional. Desta maneira podemos verificar se o prestígio profissional de uma dada profissão está linearmente relacionado ao piso salarial estimado pela sociedade.

Experimentos similares não foram encontrados na literatura. Todavia, alguns experimentos têm investigado o "Status Social" em função de vários atributos, tais como profissão (ou ocupação), renda anual, educação, raça e religião. Por exemplo, HAMBLIN \& SMITH (1966); HAMBLIN (1971) conduziu um estudo para determinar o efeito da renda anual e outras variáveis sobre "Status Social" de pessoas da comunidade e de professores universitários. Os métodos utilizados naquele estudo incluem estimação de magnitudes, estimação de razões e emparelhamentos intermodais no qual os sujeitos desenharam uma linha cujo comprimento servia para indicar a quantidade ou grau de status conferido a uma dada renda anual. Todos os três métodos produziram resultados indicando que funções de potência descrevem muito bem a relação entre status e renda anual, com expoentes variando entre 0,6 a 0,7 . Em outras palavras, o status social de uma pessoa cresce com sua renda anual, mas ele não dobra quando sua renda duplica. Na realidade

\footnotetext{
* Professor Associado junto ao Departamento de Enfermagem Geral e Especializada da Escola de Enfermagem de Ribeirão Preto da Universidade de São Paulo

** Professor Titular junto ao Departamento de Psicologia e Educação da Faculdade de Filosofia, Ciências e Letras de Ribeirão Preto da Universidade de São Paulo
} 
as respostas dos sujeitos indicaram que um aumento de $100 \%$ na renda anual confere um aumento no status social de apenas 50 ou $60 \%$. Em experimento envolvendo procedimento similar, RAINWATER (1971) obteve com o método de estimação de magnitudes do status social em função da renda anual, uma função de potência com expoente igual a 0,73 . Portanto, tomados juntos os resultados destes dois experimentos, estes mostram que a função relacionando status social a renda anual não é uma função linear, ou seja, não tem um expoente igual a unidade. Ao contrário, a relação é caracterizada por uma função linear com expoente usualmente menor que a unidade, em média igual a 0,70 .

Assim, neste Experimento verificamos se esta relação não linear, com um expoente próximo a 0,70 , permanece mesmo quando a renda (piso salarial) de diferentes profissões é subjetivamente estimada em função do prestígio que cada uma ocupa na nossa sociedade.

\section{MÉTODO}

\section{Participantes}

Participaram 20 estudantes universitários (19 femininos e 1 masculino) dos diferentes cursos de graduação do Campus da USP de Ribeirão Preto, com idades variando entre 18 e 21 anos. Todos eram ingênuos quanto aos propósitos do experimento, ou seja, nenhum deles tinha sido submetido a experimentos anteriores desta natureza.

\section{Material}

Foi elaborado um bloco de papel contendo na primeira página as instruções dadas aos sujeitos e nas páginas seguintes uma lista das mesmas treze profissões utilizadas nos experimentos anteriores.

\section{Procedimentos}

O procedimento foi idêntico aqueles empregados nos estudos anteriores envolvendo o método de estimação de magnitudes, exceto que em lugar do sujeito efetuar estimativa numérica do prestígio profissional de cada profissão, ele realizava uma estimativa do número de salários mínimos atribuídos a cada profissão. Em outras palavras, o sujeito foi instruído a assinalar um piso salarial a cada profissão que fosse proporcional a quantidade de prestígio que essa profissão possui em nossa sociedade. A profissão de Biólogo foi tomada como estímulo padrão e a ela foi designado o módulo ou valor de referência de 10 salários mínimos (SM). Dessa forma, os sujeitos deveriam estimar o piso salarial de cada uma das outras profissões, estabelecendo números de salários mínimos a cada uma delas que fossem proporcionais a quantidade de prestígio que as profissões possuem em nossa sociedade. Por exemplo, se o sujeito considerasse que uma dada profissão, em função do prestígio que ela possui, deva ter um piso salarial duas vezes maior que aquele atribuído ao Biólogo, ele deveria assinalar a ela 20 salários mínimos. Se o sujeito considerasse que uma outra profissão, em função do prestígio atribuído a ela pela nossa sociedade, deva ter metade do piso salarial do Biólogo, o sujeito deveria assinalar a ela um piso salarial de 5 salários mínimos. As diferentes profissões foram apresentadas em duas séries de treze, as quais foram dispostas em duas páginas separadas, numa ordem totalmente aleatória para cada sujeito. Cada sujeito estabeleceu 26 estimativas, sendo 2 para cada profissão.

As instruções dadas para os sujeitos requeriam que os julgamentos fossem realizados em termos do prestígio atribuído a uma dada profissão pela maioria da população. $\mathrm{O}$ experimento foi realizado num laboratório e os sujeitos fizeram os julgamentos individualmente.

\section{RESULTADOS E DISCUSSÃO}

A Tabela 1 apresenta as médias geométricas das estimativas do número de salários mínimos (ESM) e as ordenações das posições (OP) de prestígios julgadas para cada uma das profissões. Pode-se observar facilmente que a profissão de Médico recebeu maior número de salários mínimos $(\mathrm{ESM}=43,30)$, enquanto a de Biólogo $(\mathrm{ESM}=10)$ seguida pela de Enfermeiro $(\mathrm{ESM}=10,46)$ receberam os menores números de salários mínimos. Em outras palavras, o Médico ocupa a $1^{\text {a }}$ posição em prestígio profissional, enquanto a de Biólogo e Enfermeiro ocupam as posições $13^{\mathrm{a}}$ e $12^{\mathrm{a}}$, respectivamente. Do mesmo modo que nos experimentos anteriores onde o prestígio profissional foi julgado através do método de estimação de magnitudes, podemos afirmar que o número de salários mínimos atribuídos ao Engenheiro $(\mathrm{ESM}=30,97)$ é aproximadamente três vezes maior que aquele atribuído ao Biólogo $(\mathrm{ESM}=10)$. Também que o número de salários mínimos atribuídos ao Psicólogo $(\mathrm{ESM}=22,28)$ é quase que duas vezes o número de salários mínimos atribuídos aos Assistente Social $(\mathrm{ESM}=11,88)$. 
Tabela 1 - Média geométrica das estimativas dos números de salários mínimos resultantes deste Experimento (ESM) e ordenação das posições (OP) de prestígios julgadas para cada uma das profissões

\begin{tabular}{lcc}
\hline \multicolumn{1}{c}{ Profissóes } & ES M & OP \\
\hline Químico & 12,03 & $10^{\circ}$ \\
Sociólogo & 13,26 & $9^{\circ}$ \\
Dentista & 33,77 & $2^{\circ}$ \\
Engenheiro & 30,97 & $3^{\circ}$ \\
Farmac êutico & 15,69 & $7^{\circ}$ \\
Biólogo & 10 & $13^{\circ}$ \\
ENFERMEIRO & 10,46 & $12^{\circ}$ \\
Médico & 43,30 & $1^{\circ}$ \\
Físico & 13,75 & $8^{\circ}$ \\
Assistente Social & 11,88 & $11^{\circ}$ \\
Fisioterapeuta & 18,59 & $6^{\circ}$ \\
Psicólogo & 22,28 & $4^{\circ}$ \\
Fonoaudiólogo & 19,59 & $5^{\circ}$ \\
\hline
\end{tabular}

Similarmente aos estudos anteriores que envolvem estimativas de magnitudes, os dados deste experimento também sustentam a Lei de Ekman. De fato, como representada na Figura 1, a variabilidade das estimativas em número de salários mínimos, indicada pelo erro padrão da média geométrica, aumenta linearmente em função do aumento das estimativas $\left(r^{2}=0,85\right)$.

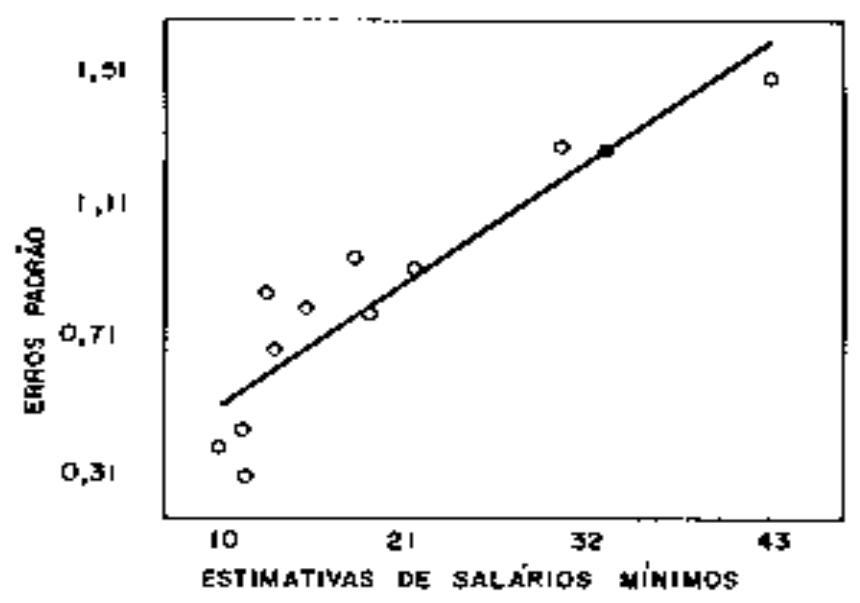

Figura 1 - Erro padrão da média geométrica em função da média geométrica das estimativas dos números de salários mínimos atribuídos a cada uma das profissões. (ESM)

Interessante comparar as estimativas numéricas do prestígio profissional de cada profissão, obtidas em cada um dos experimentos anteriores, com as estimativas do número de salários mínimos atribuídos a estas profissões em função do prestígio profissional que cada uma possui em nossa sociedade. As Figuras 2, 3, 4, 5 e 6 mostram as relações entre os logaritmos das estimativas do número de salários mínimos resultantes deste Experimento e os logaritmos das estimativas de magnitudes resultantes dos Experimentos publicados anteriormente $\left(\mathrm{EM}_{1}, \mathrm{EM}_{2}, \mathrm{EM}_{3}, \mathrm{EM}_{4} \mathrm{e} \mathrm{EM}_{5}\right)$ respectivamente, (ver Tabela 2 ). Todas as relações foram bem descritas por uma função de potência, com os expoentes sendo $0,99\left(r^{2}=0,85\right) ; 0,81\left(r^{2}=0,85\right) ; 0,74$ $\left(r^{2}=0,87\right) ; 0,70\left(r^{2}=0,84\right)$ e $0,61\left(r^{2}=0,74\right)$ para as relações entre esse estudo e os Experimentos publicados anteriormente respectivamente. Considerando-se os logaritmos das médias aritméticas de todas as estimativas de magnitudes resultantes dos Experimentos 1, 2, 3, 4 e 5 , e os logaritmos das estimativas do número de salários mínimos atribuídos a cada profissão, a relação também é caracterizada por uma função de potência com expoente igual a $0,79\left(r^{2}=0,87\right)$. Esta função está representada na Figura 7. Este valor do expoente é muito próximo aqueles observados por HAMBLIN (1971) e RAINWATER (1971), mesmo quando a relação envolve duas variáveis genuinamente subjetivas como as deste Experimento.

Tabela 2 - Média geométrica das estimativas de magnitudes resultantes de estudos publicados anteriormente $\left(\mathrm{EM}_{1}, \mathrm{EM}_{2}, \mathrm{EM}_{3}, \mathrm{EM}_{4} \mathrm{e} \mathrm{EM}_{5}\right)$

\begin{tabular}{lccccc}
\hline \multicolumn{1}{c}{ Profissões } & $\mathbf{E M}_{\mathbf{1}}$ & $\mathbf{E M}_{\mathbf{2}}$ & $\mathbf{E M}_{\mathbf{3}}$ & $\mathbf{E M}_{\mathbf{4}}$ & $\mathbf{E M}_{\mathbf{5}}$ \\
\hline Químico & 8,93 & 15,02 & 10,21 & 6,81 & 8,67 \\
Sociólogo & 7,49 & 12,36 & 9,62 & 7,74 & 6,17 \\
Dentista & 28,73 & 36,14 & 24,41 & 18,23 & 15,55 \\
Engenheiro & 27,79 & 34,73 & 22,22 & 16,60 & 14,07 \\
Farmacêutico & 13,76 & 17,38 & 14,71 & 11,71 & 11,11 \\
Biólogo & 9,87 & 13,80 & 9,95 & 8,69 & 7,96 \\
ENFERMEIRO & 11,14 & 16,23 & 12,34 & 8,86 & 8,42 \\
Médico & 34,90 & 42,80 & 29,01 & 20,91 & 19,53 \\
Físico & 9,37 & 17,13 & 9,27 & 7,13 & 8,52 \\
Assistente Social & 8,50 & 12,52 & 10,94 & 9,26 & 6,81 \\
Fisioterapeuta & 11,72 & 16,73 & 14,98 & 12,04 & 7,94 \\
Psicólogo & 17,29 & 21,15 & 16,68 & 13,92 & 9,78 \\
Fonoaudiólogo & 11,06 & 15,69 & 12,27 & 10,06 & 8,38 \\
\hline & & & & &
\end{tabular}

$\mathrm{EM}_{1}$ - Estimativas de magnitudes resultantes do estudo de Faleiros Sousa, 1993, Experimento 1

$\mathrm{EM}_{2}$ - Estimativas de magnitudes resultantes do estudo de Faleiros Sousa e Da Silva, 1996

$\mathrm{EM}_{3}$ - Estimativas de magnitudes resultantes do estudo de Faleiros Sousa, 1993 - Experimento 3

$\mathrm{EM}_{4}$ - Estimativas de magnitudes resultantes do estudo de Faleiros Sousa, 1993 - Experimento 4

$\mathrm{EM}_{5}$ - Estimativas de magnitudes resultantes do estudo de Faleiros Sousa e Da Silva, 1999 


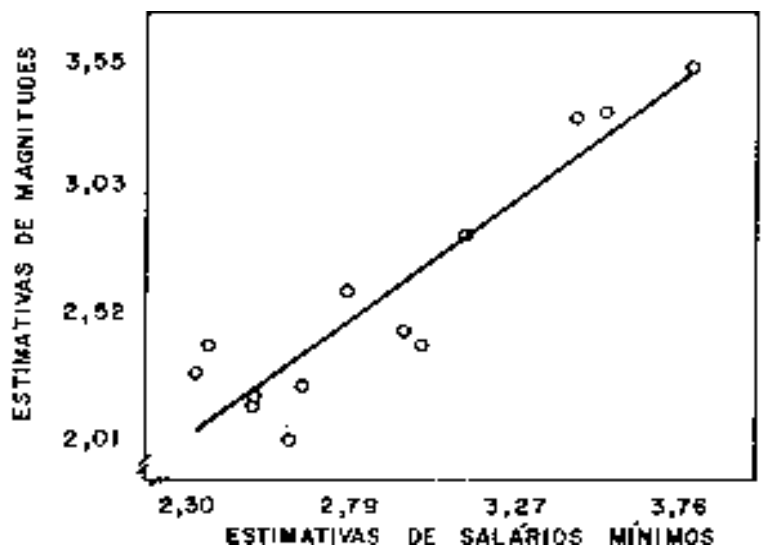

Figura 2 - Relação entre os logaritmos das estimativas de magnitudes $\left(E M_{1}\right)$ e os logaritmos das médias geométricas dos números de salários mínimos atribuídos a cada profissão resultantes deste Experimento. (EMS)

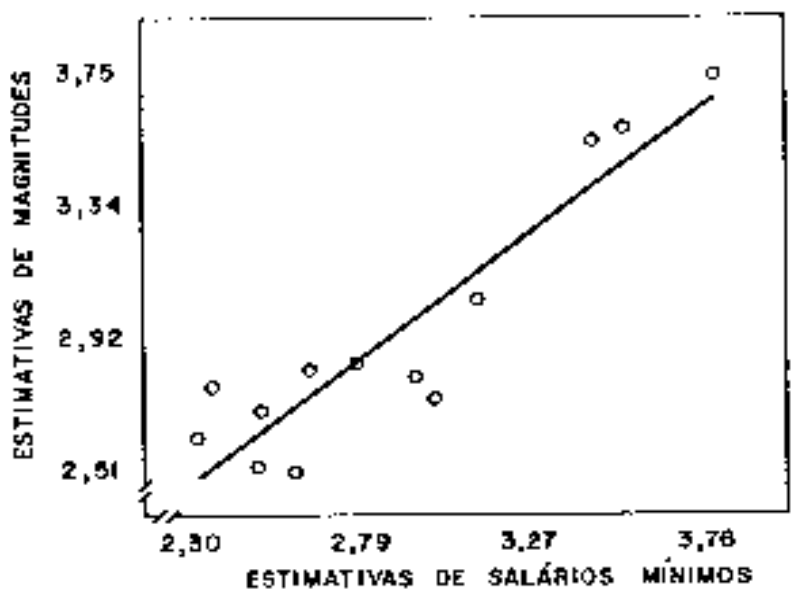

Figura 3 - Relação entre os logaritmos das médias geométricas das estimativas de magnitudes $\left(\mathrm{EMS}_{2}\right) \mathrm{e}$ os logaritmos das médias geométricas dos números de salários mínimos atribuídos a cada profissão resultantes deste Experimento. (EMS)

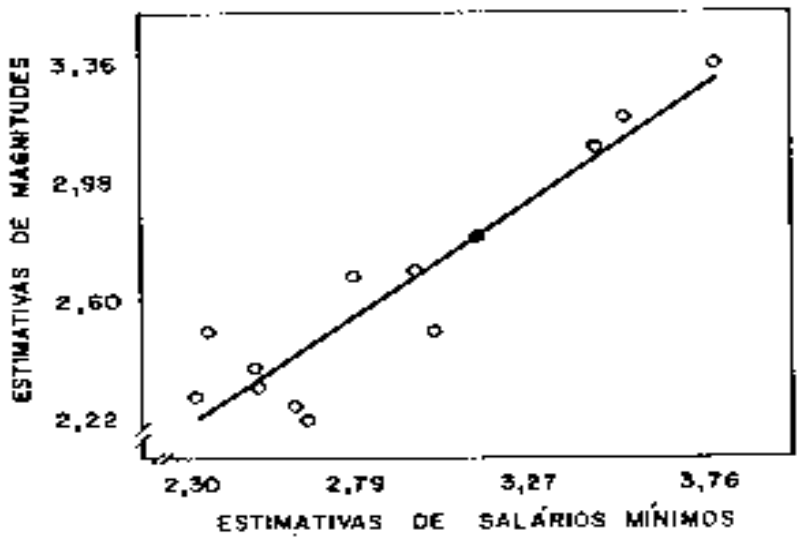

Figura 4 - Relação entre os logaritmos das médias geométricas das estimativas de magnitudes $\left(\mathrm{EM}_{3}\right)$ e os logaritmos das médias geométricas dos números de salários mínimos atribuídos a cada profissão resultantes deste Experimento. (ESM)

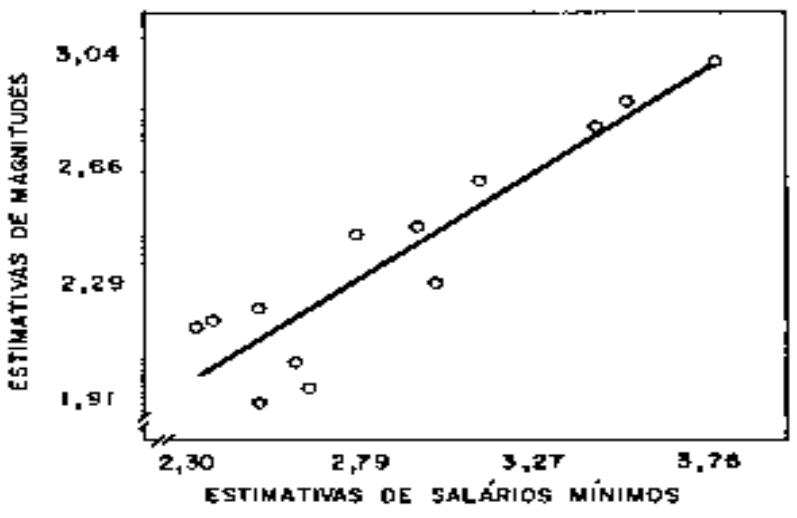

Figura 5 - Relação entre logaritmos das médias geométricas das estimativas de magnitudes $\left(\mathrm{EM}_{4}\right)$ e os logaritmos da médias geométricas dos números de salários mínimos atribuídos a cada profissão resultantes deste Experimento. (ESM)

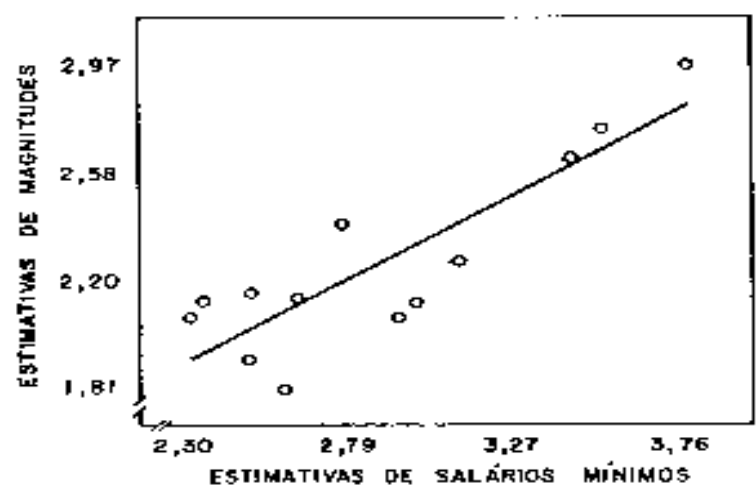

Figura 6 - Relação entre os logaritmos das médias geométricas das estimativas de magnitudes $\left(\mathrm{EM}_{5}\right)$ e os logaritmos das médias geométricas dos números de salários mínimos atribuídos a cada profissão resultantes deste Experimento. (ESM)

O coeficiente de concordância de Kendall (W) calculado entre todas as ordenações das posições de prestígios julgadas de cada uma das profissões resultantes das estimativas de magnitudes feitas nos Experimentos publicados anteriormente $\left(\mathrm{EM}_{1}, \mathrm{EM}_{2}, \mathrm{EM}_{3}, \mathrm{EM}_{4}\right.$ e EM 5 , e das estimativas do número de salários mínimos feitas neste Experimento, todas atribuídas a cada profissão, foi igual a $0,85\left(x^{2}=61,23 ; g l=13 ; p=(0,001)\right.$. Este valor indica que as ordenações dos graus de prestígios das profissões resultantes de diferentes experimentos envolvendo diferentes amostras de sujeitos são altamente concordantes entre si. Portanto, a fidedignidade destas ordenações de prestígios é substancialmente elevada, levando-se em conta que a modalidade utilizada foi a numérica (estimação de magnitudes e de salários mínimos). 


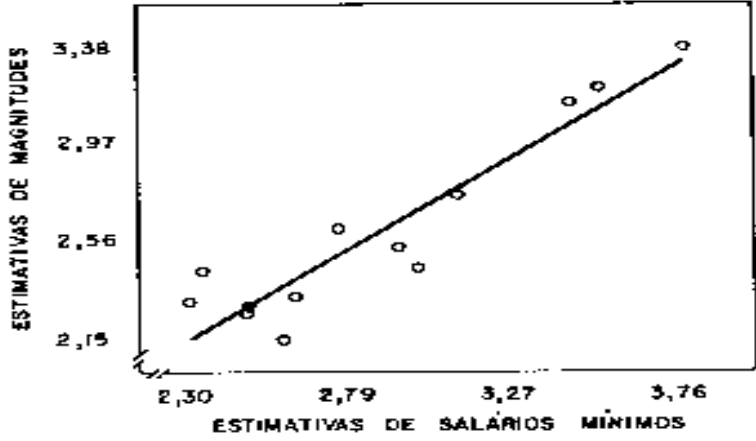

Figura 7 - Relação entre os logaritmos das médias aritméticas das médias geométricas das estimativas de magnitudes resultantes dos Estudos anteriores $\left(\mathrm{EM}_{1}, \mathrm{EM}_{2}, \mathrm{EM}_{3}, \mathrm{EM}_{4}\right.$, e EM $\left.\mathbf{E M}_{5}\right)$ e os logaritmos das médias geométricas dos números de salários mínimos atribuídos a cada profissão resultantes deste Experimento. (ESM)

\section{CONCLUSÕES}

Os dados deste experimento realizado neste estudo permitem-nos concluir que: 1 - a relação entre as estimativas de magnitudes e as estimativas do número de salários mínimos atribuído a cada profissão em função de seu prestígio é caracterizada por uma função de potência com um expoente menor que 1,0.2 - as ordenações dos graus de prestígio das profissões resultantes de diferentes experimentos envolvendo diferentes amostras de sujeitos são altamente concordantes ( $\mathrm{W}=0,85 ; \mathrm{p}<0,001)$, levando-se em conta que a modalidade utilizada foi a numérica (estimação de magnitudes e de salários mínimos).

\section{ESTIMATION OF THE NUMBER OF MINIMUM SALARIES ATTRIBUTED TO PROFESSIONS IN FUNCTION OF THEIR PRESTIGE}

The purpose of this study was to verify the relationship between professional prestige scaled through estimations and the professional prestige scaled through estimation of the number of minimum salaries attributed to professions in function of their prestige in society. Results showed: 1- the relationship between the estimation of magnitudes and the estimation of the number of minimum salaries attributed to the professions in function of their prestige is characterized by a function of potence with an exponent lower than 1,0,2 - the orders of degrees of prestige of the professions resultant from different experiments involving different samples of subjects are highly concordant $(W=0.85 ; p<0.001)$, considering the modality used as a number (estimation of magnitudes of minimum salaries).

KEY WORDS: psychophysics

\section{ESTIMATIVA DEL NÚMERO DE SALÁRIOS MINIMOS ATRIBUIDOS A CADA PROFESIÓN EN FUNCIÓN DE SU PRESTIGIO}

El propósito de este estudio fue verificar la relación entre el prestigio profesional escalonado a través de estimativas numéricas y el prestigio profesional escalonado a través de estimativas del número de salarios mínimos atribuidos a cada profesión en función de su prestigio profesional en la sociedad. Los resultados mostraron: 1- la relación entre las estimativas de magnitudes y las estimativas del número de salarios mínimos atribuido a cada profesión en función de su prestigio es caracterizada por una función de potencia con un exponente menor que 1,0,2 - las ordenaciones de grados de prestigio de las profesiones resultantes de diferentes experimentos involucrando diferentes muestras de sujetos son altamente concordantes $(W=0.85 ; p<0.001)$, considerando que la modalidad utilizada fue la numérica (estimación de magnitudes y de salarios mínimos).

TÉRMINOS CLAVES: psicofisica

\section{REFERÊNCIAS BIBLIOGRÁFICAS}

01. FALEIROS SOUSA, F.A.E. Prestígio profissional do enfermeiro: um enfoque da Psicofísica Social. Ribeirão Preto, 1993. 197p. Tese (doutorado) Escola de Enfermagem de Ribeirão Preto, Universidade de São Paulo.

02. FALEIROS SOUSA, F.A.E.; DA SILVA, J.A. Psicofísica do prestígio social: Comparação entre estimação de magnitudes e de comparação aos pares. Arquivos Brasileiros de Psicologia, v. 48, n. 3, p. 69-79, 1996.
03. HAMBLIN, R.L. Ratio measurement for the social sciences. Social Forces, v. 50, p. 191-206, 1971.

04. HAMBLIN, R.L.; SMITH, C.R. Values, status and professors. Sociometry, v. 29, p. 183-196, 1966.

05. RAINWATER, L. Interim report on social status, income and family behavior. Unpublished paper, Joint Center for Urban Studies of the Massachussetts Institute of Technology and Harvard University, 1971. 